\title{
UTILIZAÇÃO DE LEITE MATERNO EM LACTÁRIO POR BEBÊS INTERNADOS EM UNIDADES DE TERAPIA INTENSIVA
}

\section{Use of breast milk from milk dispensary by babies in neonatal intensive care units \\ Utilización de la leche materna en lactario de bebés ingresados en unidades de cuidados intensivos}

\author{
Manoella Pires Pinzon \\ Universidade de Caxias do Sul - UCS - Caxias do Sul (RS) - Brasil
}

Fernanda Oliveira Marques

Uniritter Laureate International Universities - Uniritter - Porto Alegre (RS) - Brasil

Bruna Bellincanta Nicoletto

Universidade de Caxias do Sul - UCS - Caxias do Sul (RS) - Brasil

\section{RESUMO}

Objetivo: Avaliar a quantidade de leite materno coletado e com destino à Unidade de Terapia Intensiva (UTI) Neonatal, em um hospital amigo da criança, no Sul do Brasil. Métodos: Estudo observacional, transversal e analítico, realizado no lactário do Hospital Geral de Caxias do Sul, no período de julho a dezembro de 2016. Foram coletados dados da quantidade de leite materno, considerando: entrada total para todos os setores, entrada e saída com destino à UTI Neonatal, desprezos por alta/óbito, degelo vencido e validade expirada. As variáveis categóricas estão apresentadas em números absolutos e percentuais e variáveis contínuas, como média \pm desvio padrão. Realizou-se análise estatística através do programa Statistical Package for Social Sciences (SPSS), versão 20.0, considerando significância estatística p $<0,05$. Resultados: O valor de entrada total no lactário foi de $177.053 \mathrm{~mL}$, a entrada de leite materno para a UTI Neonatal apresentou-se em $153.898 \mathrm{~mL}$, e teve como saída $87,21 \%$ desse volume. Os números de desprezo representaram 4,04, 1,14 e 0,55\%, respectivamente, sendo por alta/óbito, degelo e validade. A média diária do semestre de esgota para a UTI Neonatal foi 836,40 $3382,67 \mathrm{~mL}$, e a média diária de utilização de leite materno para a UTI Neonatal de 729,44 $\pm 341,54 \mathrm{~mL}$. Conclusão: O lactário apresentou alta porcentagem de esgota e utilização de leite materno e baixo desprezo, demonstrando bom controle de armazenamento e sua importância dentro do hospital amigo da criança.

Descritores: Leite Humano; Unidades de Terapia Intensiva Neonatal; Bancos de Leite; Extração de Leite.

\section{ABSTRACT}

Objective: To assess the amount of breast milk collected and destined for the Neonatal Intensive Care Unit (ICU) at a baby-friendly hospital in the South of Brazil. Methods: An analytical, observational cross-sectional study, carried out in the milk dispensary of Caxias do Sul General Hospital, in the period from July to December 2016. Data on the amount of breast milk was collected, considering: total intake for all hospital units, entry and exit of milk destined for the Neonatal ICU, and discarded amounts due to discharge/death, expired thaw and expired validity. Categorical variables are presented in absolute numbers and percentages and continuous variables as mean \pm standard deviation. Statistical analysis was performed using the Statistical Package for Social Sciences (SPSS), version 20.0, considering p $<0.05$ as statistical significance level. Results: The total milk intake to the milk dispensary was 177,053 mL; the breast milk entry to the Neonatal ICU was $153,898 \mathrm{~mL}$, and $87.21 \%$ of this volume was used. The disposal figures due to discharge/death, thaw and validity represented $4.04,1.14$ and $0.55 \%$, respectively. The mean daily milk expression in the semester for the Neonatal ICU was $836.40 \pm 382.67 \mathrm{~mL}$, and the mean daily consumption of human milk for the Neonatal ICU was $729.44 \pm 341.54 \mathrm{~mL}$. Conclusion: The milk dispensary presented a high percentage of human milk expresstion and consumption and a low disposal, demonstrating a good storage control and its value within the baby-friendly hospital.

Descriptors: Milk, Human; Intensive Care Units, Neonatal; Milk Banks; Breast Milk Expression. 


\section{RESUMEN}

Objetivo: Evaluar la cantidad de leche materna extraida y destinada a la Unidad de Cuidados Intensivos (UCI) Neonatal de un hospital amigo del niño en el Sur de Brasil. Métodos: Estudio observacional, transversal y analitico realizado en el lactario del Hospital General de Caxias del Sur en el periodo entre julio y diciembre de 2016. Se recogieron los datos de la cantidad de leche materna considerando la entrada total para todos los sectores, la entrada y salida para la UCI Neonatal, el desprecio por la altalel óbito, el deshielo caducado y la validez espirada. Las variables categóricas se presentan con valores absolutos y porcentuales y las variables continuas con la media \pm desviación típica. Se realizó un análisis estadístico a través del programa Statistical Package for Social Sciences (SPSS) versión 20.0 con la estadística significativa de p<0,05. Resultados: El valor de la entrada total en el lactario ha sido de $177.053 \mathrm{ml}$, la entrada de la leche materna para la UCI Neonatal ha sido de $153.898 \mathrm{ml}$ y la salida del 87,21\% de ese volumen. Los números del desprecio representaron el 4,04, 1,14 y el 0,55\%, respectivamente, por la altalel óbito, el deshielo y la validez. La media diaria del semestre de la leche agotada para la UCI Neonatal ha sido

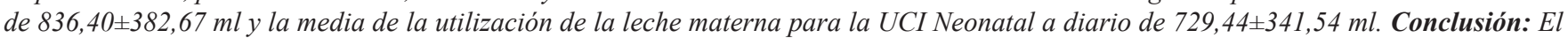
lactario presentó alto porcentaje de la leche agotada y de la utilización de la leche materna y bajo desprecio lo que señala un buen control de almacenamiento y su importancia para el hospital amigo del niño.

Descriptores: Leche Humana; Unidades de Cuidado Intensivo Neonatal; Bancos de Leche; Extracción de Leche Materna.

\section{INTRODUÇÃO}

Do ponto de vista evolutivo, nutricional e econômico, o leite humano é o alimento ideal para o bebê nos primeiros meses de vida. O aleitamento materno exclusivo (AME) nos primeiros seis meses, seguido de amamentação complementada com alimentos adequados para um ano ou mais, continua a ser o recomendado pela Academia Americana de Pediatria ${ }^{(1)}$, pelo Centro de Controle e Prevenção de Doenças ${ }^{(2)}$ e pela Organização Mundial da Saúde (OMS) ${ }^{(3)}$.

Atualmente, a duração média da AME ainda é baixa em alguns países, como em Portugal, que é de apenas 2 meses ${ }^{(4)}$. Na maioria dos países de alta renda, a prevalência é inferior a $20 \%$, tendo uma diferença importante entre o Reino Unido $(<1 \%)$ e os Estados Unidos (27\%), e entre a Noruega (35\%) e Suécia $(16 \%)^{(5)}$. Na África, no sul da Ásia e em partes da América Latina, a prevalência da amamentação até os 12 meses é a mais alta ${ }^{(5)}$. No Brasil, as taxas de AME estão em ascensão, porém continuam bem aquém do recomendado pela $\mathrm{OMS}^{(3)}$. A II Pesquisa Nacional de Prevalência de Aleitamento Materno nas Capitais Brasileiras e Distrito Federal, realizada em 2008, mostrou uma prevalência de 41\% de amamentação exclusiva entre as crianças menores de seis meses no Brasil( ${ }^{(6)}$. A duração mediana do AME em crianças menores de seis meses foi de 54 dias $^{(6)}$. Dados do ano de 2013 apontam uma prevalência de $36,6 \%$ de AME até seis meses e 52,1\% de aleitamento materno no Brasil ${ }^{(7)}$.

O leite materno (LM) é uma matriz complexa, com uma composição geral de $87 \%$ de água, 3,8\% de gordura, $1 \%$ de proteína e $7 \%$ de lactose. A gordura e a lactose, respectivamente, fornecem $50 \%$ e $40 \%$ da energia total do leite ${ }^{(8)}$. O leite da lactante de pré-termo apresenta maiores concentrações de proteína, sódio, cálcio, lipídios e particularidades anti-infecciosas ${ }^{(9)}$. Em relação às proteínas, o colostro - primeira secreção das glândulas mamárias - é rico em proteínas protetoras, especialmente a imunoglobulina secretória A, que age contra infecções e alergia alimentar. O leite maduro, por sua vez, contém mais proteínas nutritivas que o colostro (caseína e as proteínas do soro) ${ }^{(10)}$.

Uma nutrição adequada no início da vida é um fator importante para os resultados do desenvolvimento, como o neurodesenvolvimento e as habilidades cognitivas posteriores ${ }^{(11)}$. Diversas evidências apontam os benefícios do leite materno na saúde das crianças, dentre eles, uma variedade de agentes bioativos modificam a função do trato gastrointestinal e do sistema imunológico, bem como auxiliam no desenvolvimento do cérebro. Estudos comprovam que um maior volume de leite materno durante a estadia em Unidade de Terapia Intensiva Neonatal (UTIN) é benéfico para o desenvolvimento cognitivo de recémnascidos prematuros ${ }^{(12)}$. Assim, o LM é amplamente reconhecido como um fluido biológico necessário para o ótimo crescimento do bebê e seu desenvolvimento, especialmente em situação de internação hospitalar. Além disso, o LM reduz as chances de doenças metabólicas tardias, particularmente protegendo contra a obesidade e a diabetes mellitus tipo $\mathrm{II}^{(8)}$.

Uma estratégia importante é a Iniciativa Hospital Amigo da Criança para melhorar a prática do aleitamento materno. As recomendações atuais para promoção, manutenção e apoio da lactação na UTIN incluem: treinamento de pessoal específico e informações sobre os benefícios e desafios do aleitamento materno, evitando a separação mãe-bebê, promovendo interação pele-a-pele, desestimulando o uso de mamadeira, ajudando as mães a iniciar a ordenha logo depois do nascimento e orientando a utilização de esgotadeira elétrica de leite materno ${ }^{(13)}$.

A quantidade de leite materno utilizado em UTIN é importante para avaliar o funcionamento do lactário, o fluxo de esgota das mães e, principalmente, o recebimento de LM pelos bebês internados, os quais, na maioria das vezes, são prematuros. Porém ainda é um tema pouco estudado. Baseado no exposto, o presente estudo teve como objetivo avaliar a quantidade de leite materno coletado e utilizado na UTIN em um hospital amigo da criança localizado no Sul do Brasil. 


\section{MÉTODOS}

Trata-se de um estudo transversal, observacional, descritivo e analítico, onde coletaram-se os dados de entrada e de saída de LM, do lactário do Hospital Geral de Caxias do Sul, Rio Grande do Sul, Brasil, no período de julho a dezembro de 2016.

Os dados foram coletados das planilhas do setor ao longo do primeiro semestre de 2017, sendo estes: a) dados de entrada total de LM, em que se somou todo o volume coletado de LM de todas as mães que esgotaram no lactário no período e para todos os setores do hospital, sendo esse LM de mães de bebês internados na UTIN ou não; b) dados de entrada total destinada à UTIN, sendo o montante do volume de LM ordenhado somente pelas mães de bebês da UTIN; c) dados de saída do LM com destino à UTIN, referindo-se à quantidade de LM que foi ordenhado pelas mães de bebês da UTIN, foi armazenado pelo lactário e teve como fim a utilização na UTIN, não permanecendo estocado até o limite da validade, e d) dados de desprezo por data de validade expirada ( $>15$ dias congelado), por degelo vencido (> 12 horas descongelado) ${ }^{(14)}$, por alta ou por óbito. Os dados de desprezo por sujidade foram coletados, porém, não permaneceram incluídos nas estimativas, pois não constam no registro de entrada, mas em outra planilha do setor.

Em seguida, digitaram-se os dados digitados em planilha Excel. Inicialmente, somaram-se os valores diários de entrada total, entrada da UTIN, saída da UTIN, desprezo por sujidade, desprezo por data de validade expirada, desprezo por degelo vencido e desprezo por alta e óbito. As variáveis categóricas estão apresentadas em números absolutos e percentuais, e as variáveis contínuas em média \pm desvio padrão. Além disso, as variáveis estão apresentadas com resultados mensais (números absolutos) e no período total de seis meses. Para comparação entre os meses avaliados, utilizou-se teste de Kruskall-Wallis, já que a distribuição das variáveis foi não paramétrica pelo teste de Shapiro Wilk. Obteve-se a correlação entre entrada e saída de LM na UTIN por meio do coeficiente de Spearman. A análise estatística ocorreu no programa Statistical Package for Social Sciences (SPSS), versão 20.0, considerando significância estatística p $<0,05$.

O projeto recebeu aprovação pela Comissão Científica Editorial do Hospital Geral de Caxias do Sul (Protocolo 57/2015) e pelo comitê de ética da Universidade de Caxias do Sul sob Parecer n. 1.714.478.

\section{RESULTADOS}

Os valores analisados demonstram que, no período de julho a dezembro de 2016, a entrada total de LM no lactário teve um montante de $177.053 \mathrm{~mL}$. Deste total, 86,92\% (153.898 mL) eram LM de mães de bebês internados na UTIN do hospital geral. Do total de entrada de LM destinado à UTIN, obteve-se $87,21 \%(134.216 \mathrm{~mL})$ de saída, ou seja, LM levado até os bebês pelo lactário. O LM que teve entrada para a UTIN e que não teve utilização, foi desprezado pelos índices de alta/óbito do paciente, de degelo e de vencimento expirado, representando 4,04, 1,14 e 0,55\% (6.219 mL, $1.751 \mathrm{~mL}$ e $844 \mathrm{~mL}$, respectivamente) do total recebido para a UTIN. No período, $23.609 \mathrm{~mL}$ de LM foi desprezado por sujidade, tendo 131,16 mL como média diária do semestre (Figura 1).

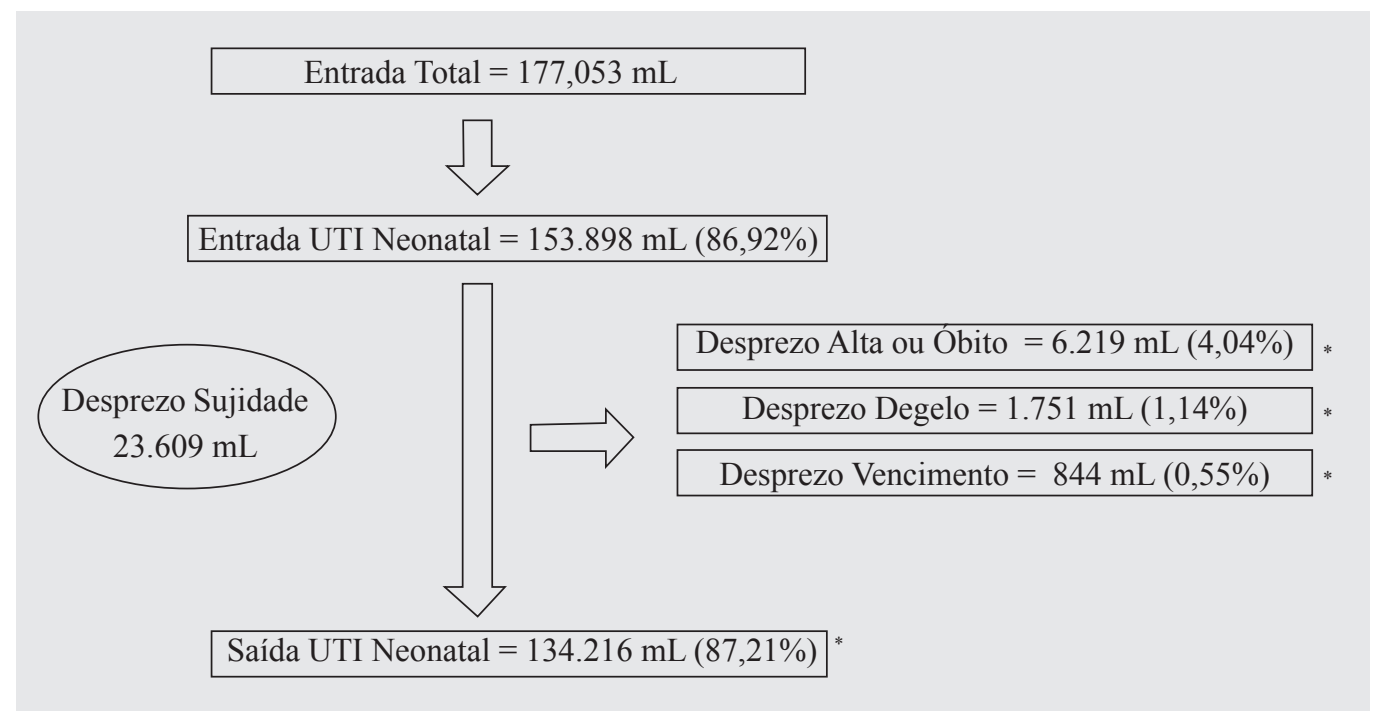

Figura 1 - Organograma de valores absolutos e percentuais de entrada, saída e desprezos de leite materno do lactário.

* As relações de entrada, saída e desprezos não totalizam 100\%, devido à coleta e utilização de um dia antes e um dia após o período da coleta de dados, já que o leite materno ordenhado pode ficar até 15 dias congelado em estoque.

* Refere-se a entrada de leite materno para UTIN - Unidade de Terapia Intensiva Neonatal (Coletado para UTIN). UTI: Unidade de Terapia Intensiva. 
A Tabela I é ilustrada por variáveis e valores mensais e a média no período durante os seis meses em que foram estudadas. Observou-se diferença entre os valores quando se comparou separadamente cada mês do período. Durante os seis meses analisados, o mês de agosto teve um volume maior de LM recebido, sendo 1360,84 mL, enquanto o mês de dezembro teve o menor, de 749,35 mL. Os meses de julho e agosto apresentaram os maiores valores de entrada total $(1230,03 \mathrm{e} 1360,84 \mathrm{~mL}$ respectivamente) e entrada UTIN $(930,97$ e $1288,48 \mathrm{~mL}$ respectivamente) quando comparados aos demais meses ( $<<0,001)$. Já na saída UTIN, o mês de julho $(777,06 \mathrm{~mL})$ foi estatisticamente igual ao mês de agosto e também igual aos outros meses, porém agosto foi estatisticamente diferente dos demais meses, apresentando o maior valor no período $(1066,94 \mathrm{~mL} ; \mathrm{p}<0,001)$.

No desprezo por degelo, julho apresenta o maior volume $(21,77 \mathrm{~mL})$ e o mês de outubro, o menor $(0,84 \mathrm{~mL})$, quando comparados aos demais meses, sendo $\mathrm{p}<0,001$. Os desprezos por alta/óbito e por vencimento não tiverem diferença estatística. As variáveis de desprezo não acompanharam o comportamento das variáveis de entrada total da UTIN e saída da UTIN proporcionalmente, pois dependem de altas e óbitos dos pacientes e da quantidade utilizada por recipiente de LM ordenhado.

Em média, entraram no lactário $962,25 \pm 423,75 \mathrm{~mL}$ de LM por dia. A média diária do semestre foi de $836,40 \pm 382,67 \mathrm{~mL}$ de LM diretamente para a UTIN (Tabela I).

Tabela I - Quantidade de leite materno apresentada em valores absolutos mensais e média diária do segundo semestre de 2016 no lactário do Hospital Geral. Caxias do Sul, Rio Grande do Sul, Brasil, 2016.

\begin{tabular}{|c|c|c|c|c|c|c|c|c|}
\hline Variável/mês & Julho & Agosto & Setembro & Outubro & Novembro & Dezembro & P valor & $\begin{array}{c}\text { Média diária do } \\
\text { semestre }\end{array}$ \\
\hline Entrada Total (mL) & $1230,03^{a}$ & $1360,84^{\mathrm{a}}$ & $767,00^{b}$ & $851,94^{b}$ & $802,87^{b}$ & $749,35^{b}$ & $<0,001$ & $962,25 \pm 423,75$ \\
\hline Entrada UTIN (mL) & $930,97^{\text {a }}$ & $1288,48^{\text {a }}$ & $717,43^{b}$ & $682,26^{b}$ & $719,17^{b}$ & $672,48^{b}$ & $<0,001$ & $836,40 \pm 382,67$ \\
\hline Saída UTIN (mL) & $777,06^{\mathrm{ab}}$ & $1066,94^{\mathrm{a}}$ & $695,87^{b}$ & $673,03^{b}$ & $596,23^{b}$ & $562,10^{\mathrm{b}}$ & $<0,001$ & $729,44 \pm 341,54$ \\
\hline Desprezo por alta/óbito (mL) & 4,84 & 124,68 & 5,67 & 2,58 & 31,07 & 32,97 & 0,286 & $33,80 \pm 215,35$ \\
\hline Desprezo por degelo (mL) & $21,77^{\mathrm{a}}$ & 7,29 ab & $11,07^{a b}$ & $0,84^{\mathrm{b}}$ & 8,93 ab & 7,23 ab & $<0,001$ & $9,52 \pm 19,41$ \\
\hline Desprezo por vencimento $(\mathrm{mL})$ & 3,97 & 4,94 & 9,60 & 0,00 & 4,17 & 5,00 & 0,363 & $4,59 \pm 22,22$ \\
\hline
\end{tabular}

UTIN: Unidade de Terapia Intensiva Neonatal; Grupos que apresentam letras diferentes são estatisticamente diferentes entre si; Utilizou-se o Teste de Kruskall-Wallis para comparação entre os meses.

Houve uma correlação positiva entre a entrada de LM para a UTIN e a saída de LM. Essa correlação foi moderada, apresentando os valores de $\mathrm{r}=0,659$ e $\mathrm{p}<0,001$, com variáveis diretamente proporcionais. Logo, quanto maior a quantidade de entrada de LM para a UTIN, maior a saída do mesmo.

\section{DISCUSSÃO}

Neste presente estudo, pôde-se observar um alto percentual de LM esgotado e também utilizado pela UTIN. A maior parte do LM esgotado pelas mães de bebês internados na UTIN foi enviado para os bebês, tendo apenas 5,73\% desse total em desprezo. Os valores de desvio padrão são altos em desprezo, pois é considerado zero na maioria dos dias, e um valor alto em um único dia, principalmente devido a altas e óbitos, em que grandes volumes de leite estocados são desprezados. Em relação aos meses do estudo, observou-se o valor aumentado nas variáveis de entrada total de LM e entrada UTIN durante os meses de julho e agosto. Possivelmente, esses valores apresentam essa diferença por haver maior número de nascimentos em relação aos outros meses estudados, ou pelas mães esgotarem em maior frequência e/ou quantidade. Os valores elevados de entrada e saíde de LM para a UTIN, assim como os baixos valores de desprezo reforçam a qualidade do lactário e sua importância na promoção de saúde. Na maioria das vezes, são bebês prematuros, que necessitam de todos os benefícios que o leite materno traz, mesmo nos casos em que não podem ainda sugar a mama, mas fazem uso desse leite ordenhado através de copinhos ou sondas, desencorajando o uso de mamadeiras.

A literatura é escassa quando se trata de quantidade de LM utilizado em UTIN e em lactários. Entretanto, existem dados referentes à entrada e saída de LM no Posto de Coleta de Leite Humano do Hospital da Criança Conceição de Porto Alegre, Rio Grande do Sul. Considerando o mesmo período do presente estudo, o posto de coleta de leite humano do Hospital da Criança Conceição obteve $90.100 \mathrm{~mL}$ de LM coletado, nos últimos 180 dias do ano de 2016, tendo como média diária do semestre $500,55 \mathrm{~mL}$. Desse total, foi distribuído $62.200 \mathrm{~mL}$ de LM, apresentando média diária do semestre de $345,55 \mathrm{~mL}$. O volume distribuído foi dividido entre 308 receptores ${ }^{(15)}$. Porém, diferentemente do presente estudo, não há dados sobre a quantidade de LM destinado à UTIN.

O Posto de Coleta de Leite Humano do Hospital da Criança Conceição de Porto Alegre teve um percentual de utilização de LM em 69\% (62.200 mL) durante o último semestre do ano ${ }^{(15)}$. Já o lactário do Hospital Geral de Caxias do Sul obteve, no mesmo período, um percentual ainda maior, alcançando 87,21\% (134.216 mL) de utilização em relação ao LM coletado, o que demonstra o alto aproveitamento de todo o leite esgotado. Da mesma forma, os valores de utilização obtidos no hospital geral foram superiores ao percentual de distribuição dos bancos de leite do estado gaúcho ${ }^{(15)}$. 
Nos 12 meses do ano de 2016, o estado obteve $5.643 .400 \mathrm{~mL}$ de leite coletado e $4.266 .600 \mathrm{ml}$ de leite distribuído, sendo proveniente de 15.651 doadoras e tendo 16.253 receptores. Utilizou-se $75,6 \%$ desse leite coletado em todo o estado ${ }^{(16)}$, o que demonstra que o lactário do hospital geral apresenta um total de utilização acima dos números encontrados em todos os bancos de leite do estado gaúcho.

O Rio Grande do Sul conta com nove estabelecimentos de banco de leite, a maioria na capital gaúcha. Um banco de leite humano é uma unidade especializada em UTIN, e uma de suas tarefas é estimular a doação de leite materno para alimentar recém-nascidos hospitalizados. Eles são responsáveis pela pasteurização e distribuição de leite humano, tendo em conta as necessidades do bebê $\hat{e}^{(17)}$.

O lactário do hospital geral de Caxias do Sul atende mães de crianças internadas que necessitem fazer a esgota das mamas. Devido a isso, a quantidade de LM que é dada entrada no setor é alta, resultante do montante de todos esses números, ou seja, todos os setores do hospital, embora o maior número represente LM retirado pelas mães dos pacientes da UTIN. A porcentagem de entrada de LM destinada à UTIN foi de $86,92 \%(153.898 \mathrm{~mL})$.

É importante para a estimulação da lactação que as mães de recém-nascidos admitidos em unidades neonatais recebam orientações adequadas e sejam encorajadas a iniciar a ordenha precocemente. Além disso, a ansiedade e a preocupação materna em relação ao $\mathrm{RN}$ pode levar ao atraso no início da expressão mamária e a inibição da ejeção de leite. Esses fatores em conjunto podem determinar insuficiência láctea ${ }^{(18)}$.

Quanto maior a quantidade de leite ordenhado, maior a quantidade de leite enviado para os bebês na UTIN, sendo eles os maiores beneficiados dessa prática. $\mathrm{O}$ aleitamento materno é o primeiro passo para a promoção da saúde de um recém-nascido. Considerada a primeira vacina do bebê, o colostro é rico em nutrientes e propriedades anti-infecciosas, que protegem o bebê através do aumento da imunidade ${ }^{(10)}$.

Manter a ordenha das mães em situações de internação hospitalar é ainda mais especial devido à oferta de proteção para esse bebê em um momento delicado como o começo da vida. A rede familiar e hospitalar deve apoiar e encorajar essa mãe a continuar a prática de ordenha para que haja maior produção de leite e seu filho receba todos os nutrientes necessários provenientes desse aleitamento ${ }^{(18)}$, e que não necessite fazer uso de fórmula infantil industrializada.

É importante que a ordenha mamária seja iniciada logo após o parto, se possível, pois a estimulação precoce das mamas, especialmente antes de 48 horas, parece ser crítica para a manutenção de produção láctea adequada nas semanas subsequentes ${ }^{(19)}$. Além do aspecto fisiológico da mãe em produzir leite para ser esgotado, vale salientar que o emocional, o desejo da mãe de amamentar e o apoio da família são essenciais para a lactação e continuidade do aleitamento materno ${ }^{(20)}$. Um estudo recente analisou o impacto do uso de leite de doadoras nas UTINs da Califórnia, e associou a disponibilidade de um banco de doadores de LM em um hospital com um aumento médio de $10 \%$ na taxa de amamentação na UTIN ${ }^{(21)}$.

Os Postos de Coleta de Leite Humano e bancos de leite realizam a coleta e armazenamento de leite, atendendo às normativas da RDC 171. Nas salas de apoio à amamentação, onde o hospital geral se enquadra, a coleta e o armazenamento do leite materno se destina exclusivamente para alimentação do próprio filho, diferente do processo de pasteurização que ocorre nos Bancos de Leite Humano ${ }^{(14)}$, que permite a utilização de LM de qualquer mãe para todos os bebês. O lactário não possui estrutura para a pasteurização do leite ordenhado, impossibilitando a utilização de LM de uma única mãe para todos os bebês internados. Se isso fosse possível, o desprezo de LM por alta/óbito não existiria, pois esse leite seria usado para alimentar outros bebês, cujas mães não têm ou não esgotam LM, ou ainda que ordenham em quantidade insuficiente para as mamadas.

A nutriz deve seguir algumas orientações prévias à coleta, tais como: fazer uso de máscara, manter cabelos presos e envoltos por touca, higienizar as mãos, braços e unhas com sabão e as mamas apenas com água morna, e secá-las com toalha descartável. Durante a ordenha do leite, é recomendado que as mães evitem conversar, e que sejam desprezados os primeiros jatos ou gotas das mamas $^{(14)}$. Não efetuando corretamente algum desses passos, principalmente a higienização das mamas, o leite ordenhado tende a ficar com sujidades a olho nu, sendo necessário desprezar o mesmo. A sujidade se dá quando há qualquer corpo estranho no leite humano, como fragmentos de pele, pêlos e fios de roupa, o que desqualifica o produto para consumo, sendo necessário o descarte de todo o conteúdo do frasco. Existe grande rotatividade de profissionais nos hospitais, o que permite períodos com técnicos sem o devido treinamento, sendo a provável causa para a persistência do resultado do descarte de leite no item de sujidade ${ }^{(22)}$.

Quanto aos valores de desprezo por sujidade no atual estudo, a média diária do semestre foi de $128,31 \mathrm{ml}$. O valor de desprezo de leite com sujidade pode melhorar com uma atenção maior na orientação de esgota, fazendo melhor higienização das mamas e intensificar treinamentos para quem orienta, salientando a importância do trabalho multidisciplinar. Os itens de desprezo são baixos, e podem ser ainda menores se a técnica de higienização de mãos, de mamas e a paramentação na sala de esgota forem feitas com maior exatidão, além de esgotar por recipiente a quantidade necessária por mamada para cada bebê, diminuindo as chances de desprezo por degelo e gerando um aproveitamento ainda maior do LM esgotado.

As recomendações para armazenamento do LM são: etiquetar o frasco com o nome da nutriz, nome do RN, data e hora da coleta, e guardar imediatamente no freezer em temperatura de $-3^{\circ} \mathrm{C}$. O leite armazenado em freezer tem validade de 15 dias e o leite que é posto para degelo deve ser totalmente utilizado dentro de 12 horas $^{(14)}$. Os desprezos por degelo maior de 12 horas e por vencimento maior de 15 dias representaram 9,52 e 4,59 $\mathrm{ml}$ de média diária do semestre, respectivamente, ambos obtendo 
pouca representatividade na atual pesquisa. O bebês podem utilizar um volume pequeno por mamada, o que também contribui para o desprezo por degelo e/ou vencimento.

Em 1990, foi implementada a Iniciativa Hospital Amigo da Criança (IHAC), um programa que mobiliza na maternidade profissionais de saúde a fazer alterações nas rotinas e condutas baseadas na conclusão dos "Dez Passos para o Sucesso do Aleitamento Materno", a fim de promover a amamentação bem-sucedida. A iniciativa qualifica profissionais para realizar avaliações e incentiva os hospitais a solicitar a acreditação ${ }^{(23)}$. A IHAC tem como objetivo informar profissionais de saúde e a comunidade e trabalhar pela adoção de leis que protejam o trabalho da nutriz. Junto ao Programa Nacional de Incentivo ao Aleitamento Materno, apóia as rotinas de serviço que promovem o aleitamento materno e que combate a livre propaganda de leites artificiais para bebês, incluindo bicos, chupetas e mamadeiras ${ }^{(24)}$.

A prática do AME proporciona à criança inúmeros benefícios, dentre eles estão: o crescimento infantil adequado, a proteção contra infecções e um melhor desenvolvimento da musculatura da cavidade bucal, trazendo efeitos positivos inclusive na inteligência. Os fatores existentes no leite materno ainda contribuem para a diminuição do risco de alergias, hipertensão arterial, colesterol elevado e diabetes mellitus, reduzindo a chance de obesidade futura ${ }^{(9)}$.

Uma limitação do presente estudo foram os dados de coleta, em que quantidades de leite podem ter sido coletadas no dia anterior do início do estudo, e quantidades utilizadas e/ou desprezadas um dia após o estudo, não sendo possível assim totalizar o valor exato de $100 \%$ nas relações de entrada, saída e desprezos do lactário.

Cada um dos pacientes recebe uma quantidade de alimento específica, com horários distintos, e cada mãe tem seu ritmo de esgota. O presente estudo não avaliou separadamente a quantidade para cada recém-nascido, tampouco quantas mamadas foram de LM, mas apenas a quantidade recebida pelo lactário e utilizada pela UTIN. Uma perspectiva para futuros estudos seria a abrangência da análise, constatando a quantidade de mães que esgotaram suas mamas e a quantidade de bebês que receberam o LM, fazendo, além de um estudo no lactário, também na UTIN, acompanhando o paciente desde a internação até o momento da alta, podendo contabilizar a quantidade de leite materno coletado e a quantidade de leite materno utilizado para cada bebê em UTIN.

\section{CONCLUSÃO}

O presente estudo conclui que o lactário analisado apresentou alta porcentagem de esgota e utilização de leite materno e baixo desprezo, demonstrando bom controle de armazenamento e sua importância dentro do hospital amigo da criança.

\section{CONFLITO DE INTERESSE}

Os autores declaram que não houve conflitos de interesse.

\section{CONTRIBUIÇÕES:}

Manoella Pires Pinzon participou do desenho do estudo, coleta de dados, análise dos dados, redação do artigo e revisão final. Fernanda Oliveira Marques participou do desenho do estudo, redação do artigo e revisão final. Bruna Bellincanta Nicoletto participou do desenho do estudo, análise de dados, redação do artigo e revisão final.

\section{REFERÊNCIAS}

1. American Academy of Pediatrics. Breastfeeding and the use of human milk. Pediatrics. 2012;129(3):827-41.

2. Centers for Disease Control and Prevention. Breastfeeding [acesso em 2017 Mar 31]. Disponível em: https://www.cdc.gov/ breastfeeding/promotion/index.htm

3. World Health Organization. Breastfeeding [acesso em 2017 Mar 31]. Disponível em: http://www.who.int/topics/breastfeeding/ en/

4. Rodrigues C, Teixeira R, Fonseca MJ, Zeitlin J, Barros H, Portuguese EPICE (Effective Perinatal Intensive Care in Europe) Network. Prevalence and duration of breast milk feeding in very preterm infants: A 3-year follow-up study and a systematic literature review. Paediatr Perinat Epidemiol. 2018;1-10.

5. Victora CG, Bahl R, Barros AJ, França GV, Horton S, Krasevec J, et al. Breastfeeding in the 21st century: epidemiology, mechanisms, and lifelong effect. Lancet. 2016;387(10017):375-90.

6. Ministério da Saúde (BR). II Pesquisa de Prevalência de Aleitamento Materno nas Capitais Brasileiras e Distrito Federal. Brasília: Ministério da Saúde; 2009. 
7. Boccolini CS, Boccolini PMM, Monteiro FR, Venâncio SI, Giugliani ER, et al. Breastfeeding indicators trends in Brazil for three decades. Rev Saúde Pública. 2017;51:108.

8. Martin CR, Ling Pei-Ra, Blackburn GL. review of infant feeding: key features of breast milk and infant formula. Nutrients. 2016; 8(5):279.

9. Sassá AH, SchmidtII KT, Rodrigues BC, Ichisato SMT, Higarashi IH, Marcon SS. Bebês pré-termo: aleitamento materno e evolução ponderal. Rev Bras Enferm. 2014;67(4):594-600.

10. Lonnerdal B, Forsum E. Casein contente of humam milk. Am J Clin Nutr. 1985;41(1):113-20.

11. Schneider N, Garcia-Rodenas CL. Early nutritional interventions for brain and cognitive development in preterm infants: a review of the literature. Nutrients. 2017;9(3):E187.

12. Lechner BE, Vohr BR. Neurodevelopmental outcomes of preterm infants fed human milk. Clin Perinatol. 2017;44(1):69-83.

13. Bonet M, Forcella E, Blondel B, Draper ES, Agostino R, Cuttini M, et al. Approaches to supporting lactation and breastfeeding for very preterm infants in the NICU: a qualitative study in three European regions. BMJ Open 2015;5(6):e006973.

14. Brasil. Agência Nacional de Vigilância Sanitária. Resolução da Diretoria Colegiada, ${ }^{\circ} 171$. Regulamento Técnico para o funcionamento de Bancos de Leite Humano, de 4 de setembro de 2006 [acesso em 2017 Mar 31]. Disponível em: https:// www20.anvisa.gov.br/segurancadopaciente/index.php/legislacao/item/rdc-171-de-4-de-setembro-de-2006

15. Rede Global de Bancos de Leite Humano. PCLH - Hospital Criança Conceição: relatórios 2017 [acesso em 2017 Maio 19]. Disponível em: rblh.fiocruz.br/pt-br/pclh-hospital-crianca-conceicao

16. Rede Brasileira de Bancos de Leite Humano. A rede brasileira de bancos de leite humano: dados estatísticos [acesso em 2017 Maio 19]. Disponível em: http://www.redeblh.fiocruz.br/cgi/cgilua.exe/sys/start.htm?sid=352

17. Meneses TM, Oliveira MI, Boccolini CS. Prevalence and factors associated with breast milk donation in banks that receive human milk in primary health care units. J Pediatr (Rio J). 2017;93(4):382-88.

18. Nascimento MBR, Issler H. Aleitamento materno em prematuros: manejo clínico hospitalar. J Pediatr (Rio J). 2004;80(5 Supl):S163-72.

19. Organização Mundial da Saúde. Evidências científicas dos dez passos para o sucesso no aleitamento materno. Brasília: Organização Pan-Americana da Saúde; 2001.

20. Cruz MR, Sebastião LT. Amamentação em prematuros: conhecimentos, sentimentos e vivências das mães. Distúrb Comun. 2015;27(1):76-84.

21. Halleux V, Pieltain C, Senterre T, Rigo J. Use of donor milk in the neonatal intensive care unit. Semin Fetal Neonatal Med. 2017;22(1):23-9.

22. Grazziotin AL, Grazziotin MC, Letti LA. Disposal of human milk donated to a human milk bank before and after measures to reduce the amount of milk unsuitable for consumption. J Pediatr (Rio J). 2010;86(4):290-4.

23. Lopes SS, Laignier MR, Primo CC, Leite FM. Baby-friendly hospital initiative: evaluation of the ten steps to successful breastfeeding. Rev Paul Pediatr. 2013;31(4):488-93.

24. Fundo das Nações Unidas para a Infância. Iniciativa Hospital Amigo da Criança [acesso em 2015 Out 23]. Disponível em: http://www.unicef.org/brazil/pt/activities_9994.htm

\section{Endereço do primeiro autor:}

Manoella Pires Pinzon

Universidade de Caxias do Sul - UCS

Rua Francisco Getúlio Vargas, 1130

Bairro: Petrópolis

CEP: 95070-560 - Caxias do Sul - RS - Brasil

E-mail: manoella_pinzon@hotmail.com

\section{Endereço para correspondência:}

Bruna Bellincanta Nicoletto

Universidade de Caxias do Sul - UCS

Rua Francisco Getúlio Vargas, 1130

Bairro: Petrópolis

CEP: 95070-560 - Caxias do Sul - RS - Brasil

E-mail: bbngehrke@ucs.br 